\title{
IMPACT OF MICROFINANCE ON THE LOCAL MICROBUSINESS
}

IMPACTO DE LAS MICROFINANZAS EN LA MICROEMPRESA LOCAL

IMPACTO DA MICROFINANÇA NA MICROEMPRESA LOCAL

Paulina Sanhuezaii

\section{Citation}

Sanhueza, Paulina (2019). Impact of microfinance on the local microenterprise. Dimensión Empresarial, 17(2). DOI: http://dx.doi.org/10.15665/dem.v17i2.1933

\begin{abstract}
Although microfinance programs were born with the specific objective of reducing poverty, currently they attend to small entrepreneurs who have limited access to traditional finance, but are not necessarily poor. These small entrepreneurs are key agents of any territory due to their capacity to create jobs, incomes and to strengthen the local entrepreneurship structure. Many studies have tried to measure the impact of microfinance programs. Preliminary results suggest that microfinance programs could be an effective instrument of local development. However, more studies have to be conducted to support this hypothesis. The objective of this work is to evaluate the impact of a microfinance program that provides microcredit on the income of microentrepreneursin the Araucanía Region in Chile. Anon-experimental, cross-sectionalstudy with a control group was carried out using thematched comparison method, using the propensityscore technique. The resultssuggest the existence of a plausible association between participating in a microfinance program and higher incomes in local microentrepreneurs. Keywords: Microfinance, local development, microcredit, microenterprises, impact assessment, Chile
\end{abstract}

\section{Resumen}

Las microfinanzas nacen con un claro objetivo de lucha contra la pobreza, pero actualmente atienden a pequeños empresarios, no necesariamente pobres, con dificultad de acceso o excluidos financieramente. Ellos son actores clave del territorio dada su capacidad de generar ingresos, empleo, y fortalecer el entramado empresarial local. Diversos estudios han intentado medir el impacto de los programas microfinancieros, sugiriendo que ellos podrían ser un instrumento efectivo de desarrollo local, sin embargo, es necesario continuar investigando en esta línea. El trabajo tiene como objetivo evaluar el impacto de un programa microfinanciero que entrega microcrédito, sobre los ingresos de microempresarios de la Región de La Araucanía en Chile. Se realiza un estudio no experimental, transversal con grupo control a través del método de matched comparison, usando la técnica propensity score. Los resultados sugieren la existencia de una asociación plausible entre participar en un programa microfinanciero y mayores ingresos en microempresarios locales. Palabras clave: Microfinanzas, desarrollo local, microcréditos, microempresas, evaluación de impacto, Chile.

\section{Resumo}

Embora as microfinanças nasçam com um claro objetivo de combater a pobreza, servindo atualmente pequenos empreendedores, não necessariamente pobres, com dificuldade de acesso ao financiamento tradicional. Eles são atoreschaveno território, dada sua capacidade de gerar renda, emprego efortalecera rede local denegócios. Váriosestudostentaram mediro impacto dos programas de microfinanças. Os resultados preliminares sugerem que eles poderiam serum instrumento eficaz de desenvolvimentolocal, noentanto,énecessáriocontinuarinvestigandonestalinha. Oobjetivo destetrabalhoéavaliar o impacto de um programa de microfinanças que oferece microcrédito aos rendimentos de microempresários na Região de Araucanía, no Chile. Um estudo transversal não experimental, com um grupo controle, foi realizado utilizando o método de matched comparison, utilizandoa técnica de escore de propensityscore. Os resultados sugerema existência deumaassociação plausível entre a participação em um programa de microfinanças e maiores rendimentos em microempresários locais. Palavras-chave: Microfinanças, desenvolvimento local, microcréditos, microempresas, avaliação de impacto, Chile. 


\section{INTRODUCCIÓN}

El desarrollo endógeno local se define como un proceso de crecimiento económico y cambio estructural, cuyo principal objetivo es el logro en la calidad de vida de la población local, a través de la participación de diversos recursos y actores del territorio, entre los cuales destacan la micros, pequeñas y medianas empresas (Vázquez Barquero, 1999, 2013; Allende, 2000; Boisier, 2001; Alburquerque, 2002, 2007; Vázquez Barquero y Rodríguez Cohard, 2018). De tales unidades productivas, desde hace años se ha considerado que la microempresa hace una contribución importante al empleo, a la producción y al ingreso nacional en América Latina y el Caribe, y a la promoción del talento, fortaleza y flexibilidad empresarial para hacer frente al dinamismo de la economía mundial (BID, 2005; Angelelli, et al., 2006; Torres, 2006; Váldes y Sánchez, 2012; Dini y Stumpo, 2018).

De acuerdo a García (2004), Foschiatto y Stumpo (2006) y Lacalle (2008), uno de los principales problemas que aún siguen enfrentando estos actores económicos es el acceso a financiación. Tal problemática se resuelve en el bloqueo de proyectos que, en muchos casos, podrían ser exitosos, y su amplio reconocimiento ha dado lugar al ensayo de una alternativa de financiación a nivel mundial bajo el concepto de las microfinanzas. Ellas engloban una serie de instrumentos financieros de apoyo a sectores excluidos del ámbito financiero tradicional. EI microcrédito es uno de estos productos, destacando por ser el más conocido, difundido y ofertado. En la actualidad, de acuerdo con Martín (2007) y Rosenberg (2010), es posible identificar la existencia de una industria microfinanciera, con gran cantidad de instituciones dentro de ella a diferentes escalas y ofreciendo una diversidad de productos financieros.

De lo anterior se desprende que las microfinanzas pueden jugar un rol importante en el desarrollo local. De hecho, se han convertido en los últimos años en uno de los instrumentos más frecuentes incorporados por las políticas de promoción del desarrollo (Bercovich, 2004; Mballa, 2017). Por un lado, contribuirían a reducir la pobreza y la exclusión social en el territorio, permitiendo que sectores marginales generen actividades económicas y autoempleo, y por otro, favorecerían la creación de puestos de trabajo en pequeñas empresas locales, así como la propia supervivencia de dichas empresas (y empleos).

Todo lo anterior constituye la motivación para la realización de la presente investigación, donde determinar si las microfinanzas pueden ser un instrumento de desarrollo local, es un tema de gran importancia dados los bajos y medios niveles de desarrollo que ciertas economías mantienen, a pesar de las políticas implementadas en torno a una creciente globalización. Estas dinámicas son factibles de responder mediante una evaluación de impacto, un enfoque que mide los resultados de la intervención de un programa aislándolo de otros posibles factores.

Si bien a nivel mundial se han llevado a cabo diversos estudios que han intentado evaluar los impactos generados por los servicios microfinancieros (Sebstad y Chen, 1996; Goldberg, 2005; Odell, 2010, 2015) los resultados suelen ser alentadores, pero aun poco claros (Banco Mundial, 2008), centrados generalmente en economías asiáticas en vías de desarrollo, con rigor y metodologías que varían ampliamente (Dunford, 2006). Lo anterior hace necesario continuar investigando y desarrollando estudios de impacto de rigurosidad, que provean evidencia necesaria para establecer el real aporte de las microfinanzas no solo en territorios caracterizados por altas tasas de pobreza y exclusión social, sino también en aquellos de mayor nivel de desarrollo, en los que ellas pueden jugar un rol importante en la creación y fortalecimiento del tejido productivo local.

Para ello este estudio ha tomado como unidad de análisis a la economía chilena, específicamente a la Región de La Araucanía, dadas las características 
que el país y la región reúnen para un análisis dentro del marco del desarrollo local endógeno. Esto es, una economía de desarrollo medio elevado en comparación a otras economías de la región, caracterizada por una clara concentración y desigualdad económica y social entre sus regiones (PNUD, 2018). De hecho, en mayo del 2010 Chile completó el proceso a través del cual pasaba a ser oficialmente miembro de la Organización para la Cooperación y el Desarrollo Económico (OCDE), constituyéndose así, en el primer país de América del Sur en formar parte de este grupo de economías. Sin embargo, aún existen territorios que presentan elevados niveles de pobreza y exclusión (Ministerio de Desarrollo Social, 2018; PNUD, 2018). De esta forma, una investigación en Chile, y concretamente en La Araucanía, región en la cual no existen suficientes estudios de este tipo, se constituiría en un aporte a la evidencia de las virtudes de las microfinanzas en distintos contextos socioeconómicos.

Como objetivo, la investigación se plantea evaluar el impacto de las microfinanzas como instrumento financiero de promoción del desarrollo local del territorio, mediante su rol social (disminución de la pobreza) y su rol económico (fortalecimiento productivo empresarial), llevando a cabo para ello una evaluación de impacto que permita cuantificar la aplicación de un programa microfinanciero (que conceda microcréditos) en los ingresos de

\section{REVISIÓN DE LA LITERATURA}

Microfinanzas y microcrédito: definición y potencial aporte al desarrollo local

Antes de establecer los potenciales aportes de las microfinanzas en general, y del microcrédito en particular, al desarrollo local, es necesario abordar una definición para tales conceptos, ya que, tal como señalan Lacalle, Rico, Márquez y Durán (2006), se distinguen diversas prácticas que complican la microempresarios locales beneficiarios del programa. Para ello, se implementa un modelo de evaluación de impacto, a partir de una investigación no experimental, de corte transversal, en la cual se comparan dos grupos (uno que ha sido beneficiario de microcrédito, y otro que no lo ha sido, constituyéndose este último en el grupo control o contra-factual) a través del método de matched comparison (emparejamiento o comparación pareada) y la técnica propensity score, para lo cual se estimarán modelos logísticos binarios que permitirán el emparejamiento final entre un beneficiario de microcrédito y un control. Los resultados de las diferentes estimaciones calculadas permiten establecer una asociación plausible entre la participación de microempresarios de La Araucanía en un programa microfinanciero regional (concretamente un programa microcrediticio) y tener un mayor nivel de ingresos.

El artículo se estructura en cuatro secciones: en primer lugar, el marco teórico desarrolla el concepto de microfinanzas y de evaluación de impacto, en conjunto con las principales evaluaciones de impacto en el ámbito microfinanciero, desarrolladas a nivel mundial; en segundo lugar, la sección metodológica detalla el diseño, muestra, fuentes de información y procedimientos de análisis de la información en la investigación; en la sección tercera, se presentan los resultados, referentes a la cuantificación de la evaluación de impacto diseñada, y; finalmente, la cuarta sección, muestra las conclusiones e implicancias de la investigación.

generalización de estos, producto de los diversos contextos socioeconómicos y culturales entre Latinoamérica, África, Asia, EEUU y Europa. En primer lugar, es necesario realizar una distinción entre los conceptos. Así, las microfinanzas engloban una serie de instrumentos financieros (cuentas de ahorro, seguros, planes de pensiones, entre otros) de apoyo a sectores que quedan excluidos del ámbito financiero 
tradicional; y el microcrédito, por su parte, es uno de estos instrumentos (Berezo, 2005; Lacalle, et al.., 2006).

En segundo lugar, cabe concretar un poco más ¿qué es el microcrédito? La Primera Cumbre Global de Microcrédito realizada en Washington D.C. en febrero de 1997, establece que los microcréditos son Programas de concesión de pequeños créditos a los más necesitados de entre los pobres para que éstos puedan poner en marcha pequeños negocios que generen ingresos con los que mejorar su nivel de vida y el de sus familias (Lacalle, 2001, p. 125). La Comisión Europea (2003, p. 70) lo define como el instrumento de financiación más extendido para fomentar el desarrollo local. Previamente y en el mismo sentido Clark y Kays (1995) afirman que es un préstamo muy pequeño ofrecido a la microempresa, y no a personas pobres. Es en este último concepto donde se liga el microcrédito a la microempresa, centrándose en el uso empresarial que se le da a éste.

A pesar de la diversidad de definiciones, resaltan las condiciones de instrumento de financiación, instrumento de lucha contra la pobreza e instrumento de desarrollo local que abarcaría el concepto. Pero concretamente, ¿cómo puede el microcrédito, como principal servicio ofrecido por las microfinanzas, aportar al desarrollo local del territorio? Al respecto, hay que especificar que a través de la generación de facilidades en el acceso a estos recursos, es posible potenciar y fortalecer iniciativas productivas de pequeña escala (microempresas), las cuales juegan un papel determinante dentro de una estrategia de desarrollo local, al ser uno de los actores económicos del desarrollo, poseedores de recursos humanos (microempresario y trabajadores) y de recursos económicos (microempresa), pero con grandes dificultades de acceso a recursos financieros (entre ellos el crédito) (BID, 2005; Lacalle, 2008; Rojas, 2017; INE, 2015, 2017; OCDE, 2012, 2018).

Es en este aspecto donde resalta la importancia de la concesión de microcréditos. Su disponibilidad facilitaría el desarrollo de estas unidades económicas, y con ello se permitiría alcanzar tres objetivos en términos de desarrollo local: primeramente un objetivo de eficiencia, a través de la potenciación, fortalecimiento y desarrollo de microempresas, es decir, del entramado productivo de un territorio, a través de una visión en la cual los propios actores del territorio participan en su crecimiento; en segundo lugar, un objetivo de equidad a través de la participación de microempresarios excluidos tradicionalmente de procesos productivos locales debido a sus escasas posesiones y garantías que les imposibilitaba el acceso a los sistemas financieros tradicionales para obtener el capital necesario que les permitiese poner en marcha sus ideas; $y$, finalmente, si la concesión del crédito se enfoca al desarrollo de unidades microeconómicas eficientes, responsables y respetuosas con el medio ambiente, se estaría avanzando en la senda de un desarrollo ambiental sostenible.

\section{Evaluación de impacto: concepto y evidencia en el sector de las microfinanzas}

De forma concreta, y en concordancia con lo planteado anteriormente, ¿cómo podrían evaluarse los verdaderos y reales efectos de un programa microfinanciero?, ¿cómo podría estimarse el impacto sobre sus beneficiarios? A tal pregunta da respuesta la implementación de una evaluación de impacto. Según lo establecido por Karlan y Goldberg (2007), la importancia de llevar a cabo evaluaciones de impacto en el campo de las microfinanzas está respaldada en a lo menos cuatro razones: factibilidad de diseñar mejores productos y procesos a partir del mejor conocimiento que se genera sobre los clientes; comparar el costo de mejorar las condiciones de vida de las familias receptoras del microcrédito con el costo de generar el mismo impacto a través de otras intervenciones; provisión de información sobre productos y servicios que funcionarían de mejor forma para determinados tipos de clientes; y aprendizaje de las 
estructuras alternativas en la atención microfinanciera y de su influencia en el impacto de los clientes.

Se entiende por evaluación de impacto al análisis que permite determinar cuál es el cambio que se ha generado en los participantes de un programa, proyecto o política, en comparación a una situación en la que no hayan participado (Hulme, 2000; Karlan y Goldberg, 2007, Odell, 2015). En dicha definición queda expuesta la principal problemática de tales evaluaciones: la construcción del escenario alternativo o contrafactual. Es decir, el grupo de individuos que no ha participado del programa, ya que los beneficiarios no pueden, simultáneamente, haber y no haber participado en él. En el caso concreto de las evaluaciones de impacto de programas microfinancieros, por ejemplo, en la concesión de microcrédito, se intenta evaluar los cambios en la vida de los individuos beneficiarios de microcréditos identificando que, en primer lugar, la cadena de impacto se origina con la entrega del microcrédito a un individuo sobre el cual se generarán ciertos cambios. En segundo lugar, que dichos cambios podrían producirse en diferentes niveles: individual, familiar, microempresarial, comunitario e institucional. $\mathrm{Y}$, finalmente, que los posibles indicadores capaces de dar cuenta del impacto generado por ser beneficiario de microcrédito, en cada uno de los niveles anteriores, podría ser del tipo económicos, sociales, sociopolíticos y psicológicos (Hulme, 2000; SEEP Network, 2001; Lacalle y Rico, 2007).

Ahora bien, la experiencia en torno a la evaluación de la eficiencia de los programas microfinancieros de acuerdo con el Banco Mundial (2008), si bien es alentadora, en general es poco clara, ya sea porque tienden a ser estudios de poca rigurosidad metodológica, o bien porque son estudios parciales. Establecer de forma concreta su eficiencia, requiere de estudios empíricos que de forma rigurosa establezcan los impactos, y tales estudios aún son insuficientes (Gutiérrez, 2000; Lacalle, 2008;
Rosenberg, 2010), sin embargo, conforman un sustento inicial al planteamiento de que constituyen un instrumento potencial de desarrollo local.

Los estudios y recopilaciones de estudios de Berger y Buvinic (1989), Hulme y Mosley (1996), Mustafa (1995), Sebsad y Chen (1996), Barnes (1996), Todd (1996), McNelly y Dunford (1999), Coleman $(2002,2006)$,

Littlefield (2003), Menon (2003), Khandker (2005), Dunn (2005), Goldberg (2005), Karlan y Valdivia (2006), Rico, Lacalle, Durán y Márquez (2006), Kondo (2007), Duflo, Crepón, Parienté y Devoto (2008), Rico (2009), Benerjee, Duflo, Glennerster y Kinnan (2010), Allen, Armendáriz, Karlan y Mullainathan (2010), Crépon, Devoto, Duflo y Parienté (2011); Metcalfe, Gash, Gray, Reinsch, Chandler y Dunford (2012), Banerjee (2013), Cull, Ehrbeck and Holle (2014), Angelucci, Karlan and Zinman (2015), Attanasio et al. (2015), Augsburg et al. (2015), Banerjee, Karlan and Zinman (2015), Crepon et al. (2015), entre otros, arrojan resultados que indican aumentos en los niveles de ingreso, de ahorros, de salarios, en el ahorro de microempresarios y sus familias, mejoras en el acceso a salud, educación y otros servicios sociales de las familias, mejoras en el acceso al mercado crediticio; igualmente, que logran un mayor empoderamiento de la mujer, potencian aptitudes innovadoras y comportamientos de solidaridad y colaboración. En general, y hasta el año 2005, la metodología ha consistido en estudios observacionales con uso de grupo control. La idea ha sido comparar una muestra de clientes con una de noclientes, conformando este último grupo a través de individuos no-clientes seleccionados para el programa pero que finalmente no terminaron participando en él, o individuos no-clientes que podrían haber participado pero que no se vieron beneficiados debido a que vivían en villas vecinas en las que no funcionaba el programa (Goldberg, 2005). Es a partir del año 2005 cuando se observa un incremento en estudios con metodologías experimentales, las cuales se han intensificado en los últimos años, con 
la incorporación de nuevos servicios financieros para los cuales se quiere estudiar su eficiencia, y también nuevas variables o procesos sobre los cuales se desea evaluar impactos. (Odell, 2010, 2015). Aunque en muchos casos las bases metodológicas son limitadas y cuestionadas, la evidencia apunta a resultados positivos, aunque modestos, en microempresarios, en sus familias y en la comunidad a la que pertenecen, pero claramente es necesario avanzar en la cantidad y calidad de las investigaciones para concluir efectos concretos, y evaluar de tal forma si las microfinanzas son eficientes en el fortalecimiento microempresarial y en la consecución de desarrollo local. La evidencia ha tenido fuerte énfasis en las experiencias de países asiáticos, destacando Bangladesh, y se está

\section{METOdOLOGÍA}

\section{Diseño del estudio}

El diseño de estudio corresponde a una investigación no experimental (estudio de observación o estudio de no manipulación), de corte transversal en el que se compara a dos grupos: uno que ha sido beneficiario de microcrédito, y otro que no lo ha sido, constituyéndose este último en el grupo control. La construcción de los grupos comparables más parecidos estadísticamente se realiza a través del método de matched comparison (emparejamiento o comparación pareada), usando la técnica propensity score, a través de la estimación de un modelo de regresión logística. Dicho modelo usa como variable explicada una del tipo discreta binomial, que señala la positiva o negativa recepción de un microcrédito, y como variables explicativas a características del microempresario, de su hogar y de su microempresaiii, tales como: género, edad, estado civil, educación, si este(a) es cabeza de familia, tamaño del hogar, localización de la microempresa, sector al que pertenece la microempresa y tamaño de la microempresa (medido en número de trabajadores).

Para el emparejamiento se prueba con diferentes alternativas, con el fin de generar el individuo control completando con estudios llevados a cabo en otras partes del mundo (Dunford, 2006, Odell, 2015). Sin embargo, el rigor y la calidad de ellos ha variado ampliamente, impidiendo establecer resultados concluyentes, lo cual hace necesario continuar investigando y desarrollando estudios de impacto de calidad y rigurosidad científica que provean evidencia necesaria para establecer el real aporte de las microfinanzas en sus beneficiarios, no sólo en economías caracterizadas por altas tasas de pobreza y exclusión social, sino también en aquellas economías de mayor nivel de desarrollo en las que ellos juegan, tal como previamente la investigación ha planteado, un papel fundamental en la creación y fortalecimiento de tejido productivo protagonista del desarrollo local.

más parecido a su beneficiario correspondiente, y así reducir posibles sesgos de selección producto de variables observables. Concretamente se llevan a cabo dos tipos de emparejamiento: Tipo I, en el que el ingreso de cada beneficiario con probabilidad " $x$ " se empareja con el promedio de los ingresos de los individuos controles que presentantal probabilidad " $x$ "; $y$ Tipoll,enelqueel promedio de los ingresos de los beneficiarios con probabilidad " $x$ " se empareja con el promedio de los ingresos de los controles con idéntica probabilidad estimada " $x$ ".

Finalmente, el impacto del microcrédito sobre los ingresos de los beneficiarios del programa se estima calculando el promedio del impacto para cada pareja de beneficiariocontrol, comparando el ingreso promedio de las personas que recibieron microcrédito, con el ingreso promedio de las personas "similares" que no recibieron microcrédito.

\section{Muestra}

Se aplicó un muestreo bietápico por conglomerado, seleccionando, en primer lugar, instituciones 
oferentes de microcrédito heterogéneas en cuanto al mercado atendido. Concretamente, los conglomerados se constituyen por instituciones con fines de lucro e instituciones sin fines de lucro (banca y ONG, respectivamente). Se seleccionó al menos una institución representante de cada conglomerado, y posteriormente se solicitó a cada institución generar una muestra aleatoria de los microempresarios atendidos por cada una de ellas.

Así, la composición de la muestra total consta de 1279 observaciones: 407 microempresarios beneficiarios de microcréditos, distribuida en dos conglomerados (uno correspondientea microempresariosatendidos porlabanca y otro por ONG, que en el año 2007 ya habían participado de un programa microcrediticio), y 872 microempresarios no beneficiarios de microcréditos (grupo control), la cual se obtiene de una fuente secundaria con representatividad regional (Encuesta de Caracterización Socioeconómica de los Hogares de Chile, Casen).

\section{Análisis de la información}

El análisis se lleva a cabo mediante el uso del paquete estadístico SPSS. Así, siguiendo las recomendaciones de Aroca y Hewwings (2009), el análisis se estructura en 2 partes: Análisis Conjunto, el cual trabaja con la muestra total de microempresarios beneficiarios de microcrédito, procedentes del banco y la ONG regionales; y Análisis Independiente, el cual trabaja de forma independiente la muestra de beneficiarios del banco y la muestra de beneficiarios de la ONG, regionales. Al mismo tiempo, cada análisis se lleva a cabo para 3 tipos de muestras diferentes del grupo de control: Muestra №1, compuesta por empleadores, trabajadores por cuenta propia y empleados; Muestra №2, compuesta por empleadores y trabajadores por cuenta propia; y Muestra №3, compuesta por empleados. Esto se debe a que es necesario obtener un grupo control que cumpla con dos requisitos: en primer lugar, que sean individuos microempresarios $y$, en segundo lugar, individuos que no hayan sido beneficiarios de microcrédito. Al no existir una base de datos oficial de la cual se pueda extraer dicha información, es necesario generarla sobre la base de ciertos supuestos. Se utilizó para ello la Encuesta Casen, sin embargo, dicha encuesta no proporciona información directa de qué individuos cumplen con los dos requisitos establecidos previamente.

Tan sólo permite identificar individuos empleadores o trabajadores por cuenta propia de una empresa del tipo micro (1 a 9 trabajadores), o individuos empleados en una microempresa. Con la primera base de datos se obtienen microempresarios (o individuos con capacidad emprendedora), pero no se asegura el que éstos no hayan sido beneficiarios de microcrédito (a pesar de que existe una pregunta que permite identificar a aquellos individuos que no tienen deuda, ello no significa necesariamente que en el pasado cercano no hayan sido beneficiarios de microcrédito). Dado lo anterior, y de manera tal de reducir o minimizar dicho problema se selecciona una segunda base de datos compuesta por empleados de una microempresa, con la cual nos aseguramos de que los individuos no han sido beneficiarios de microcréditos (pues no son emprendedores de una microempresa), sin embargo, sabemos que no son microempresarios y que podrían no presentar características asociadas a un emprendedor. Dado lo anterior, se podría estar subestimando o sobreestimando el impacto del microcrédito, respectivamente. Por ello se realiza un análisis por separado para cada base de datos del grupo de control, y un análisis conjunto que promediaría, y con ello minimizaría, los errores de estimación de tales impactos.

Finalmente, cada análisis anterior se lleva a cabo con los dos tipos de emparejamientos ya mencionados previamente. 


\section{RESULTADOS}

\section{Medición del impacto}

El impacto del microcrédito en los individuos beneficiarios se resume en la Tabla 1 para cada tipo de análisis. Se observa así que 12 de las 18 estimaciones generan resultados esperados, es decir, resultados en los que los beneficiarios de microcrédito presentan ingresos más altos que los individuos que no han recibido microcrédito. Por otra parte, tan solo 8 estimaciones de impacto resultan estrictamente significativas, viéndose dichos resultados reducidos a 5 si se quiere analizar solo para los que muestran resultados esperados.

Al llevar a cabo el Análisis Conjunto, se observan resultados significativos, sin embargo, en los Análisis Conjuntos №1 y №2, el diferencial de medias de los ingresos es a favor de los no beneficiarios de microcrédito. Por el contrario, el Análisis Conjunto №3 muestra diferenciales en las medias de los ingresos en torno al $13 \%$, con cada tipo de emparejamiento, y a favor de los beneficiarios, sin embargo, su significancia es menor que la de los análisis previos.
Tales resultados podrían explicarse, en primer lugar, tomando en cuenta que la muestra total de beneficiarios es heterogénea, con lo cual, se estarían promediando los ingresos de individuos muy diferentes, que en general tienden a mostrar distintas medias. En segundo lugar, al trabajar con muestras diferentes del grupo control, también se promedian ingresos de individuos empleadores y trabajadores por cuenta propia, con individuos empleados, lo que resulta finalmente en que, al quitar de dicha muestra a los primeros, los ingresos medios bajen, generando un resultado positivo en el análisis №3. Lo anterior se corrobora al ver que el ingreso promedio de los beneficiarios del banco es alrededor de un $250 \%$ más elevado que el de los de la ONG, sugiriendo que banco y ONG atienden sectores muy distintos en términos de sus ingresos. Así, estudiar por separado a estas muestras podría tener mayor pertinencia.

Tabla 1. Impacto del microcrédito en ingresos de microempresarios, La Araucanía-Chile

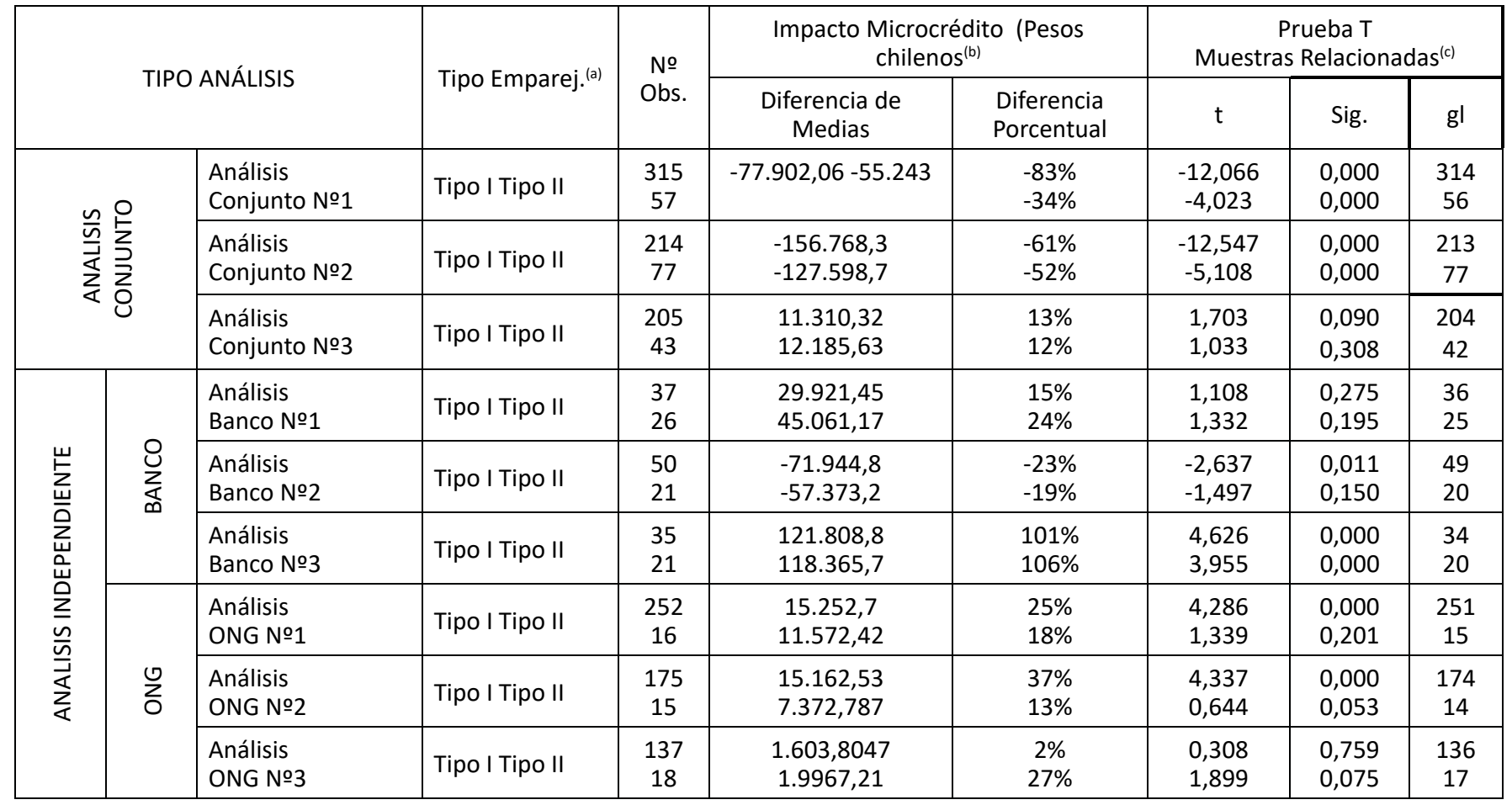

Notas: (a) Emparejamiento Tipo I: el ingreso de los beneficiarios con probabilidad " $x$ " se empareja con el promedio de los ingresos de los individuos controles que presentan dicha probabilidad " $x$ "; Emparejamiento Tipo II: el promedio de los 
ingresos de los beneficiarios con probabilidad " $x$ " se empareja con el promedio de los ingresos de los individuos controles que presentan idéntica probabilidad estimada " $x$ ". (b) Medido como el promedio del diferencial de ingreso de los beneficiarios de microcrédito y los no beneficiarios de microcrédito. (c) Para un nivel de confianza del 95\%. Fuente: Elaboración propia.

Al analizar el impacto sobre los microempresarios beneficiarios del banco, se obtiene que el microcrédito genera un impacto positivo en los microempresarios del Análisis №1 en torno al $15 \%-25 \%$, pero no significativo; genera un impacto positivo y significativo, en torno al $100 \%$ en los microempresarios para el Análisis №3; y genera un impacto negativo en torno al $20 \%$ en el Análisis №2, no significativo. En el caso en el cual el impacto resulta más favorecedor para los microempresarios (Análisis №3), se tiene en cuenta que podría estarse sobreestimando el efecto del microcrédito, ya que la muestra del grupo control compuesta por empleados, podría estar entregando información de individuos que, si bien no han recibido microcréditos, no necesariamente podrían cumplir con todas las cualidades y capacidades para ser microempresarios, concluyendo ello, probablemente, en ingresos más bajos para este colectivo. Por otra parte, la situación en la cual el impacto resulta no favorecedor (Análisis №2), podría estar subestimando el efecto en los beneficiarios, ya que al estar compuesto el grupo control por individuos del tipo empleadores y trabajadores por cuenta propia, se cumple con el requisito de reunir individuos con capacidades empresariales, pero no hay certeza de que no hayan sido beneficiarios de microcréditos.

\section{CONCLUSIONES}

Los resultados permiten establecer una asociación significativa entre la participación de microempresarios de la Región de La Araucanía en un programa microfinanciero regional (concretamente un programa microcrediticio) y tener un mayor nivel de ingresos. Lo anterior constituye una evidencia adicional del potencial de este instrumento en el combate de los niveles de pobreza y en el fortalecimiento de la estructura productiva más débil de las localidades.
Para los beneficiarios del programa de la ONG, en tanto, se obtienen impactos positivos del microcrédito en los ingresos, dicho impacto suele ser menor que en el caso de los clientes del banco, sin embargo, tiende a ser más estable y significativo. Específicamente: el Análisis №1 muestra un impacto positivo en torno al 25\%; el Análisis №2 muestra un impacto positivo en torno al 37\%, y el Análisis №3 muestra un impacto positivo en torno al $27 \%$. Nuevamente, cabe distinguir en las estimaciones de cada muestra, que el trabajar con grupos separados puede hacernos incurrir en una probable sub y sobreestimación, que debe ser considerada a la hora de interpretarse los resultados.

Por tanto, el análisis por separado de los beneficiarios del banco y de la ONG tiende a mostrar mejores resultados. En concreto, 10 de 12 estimaciones arrojan resultados de acuerdo con los resultados esperados; es decir, resultados que muestran que los ingresos de los beneficiarios de microcrédito son mayores a aquellos individuos no beneficiarios. Y, de tales estimaciones, 6 son altamente significativas. Así, los resultados permiten establecer una asociación significativa entre la entrega de microcrédito y la generación de mayores ingresos en los beneficiarios de dichos programas locales.

Si bien los resultados anteriores son alentadores, el microcrédito debe ser considerado como parte de un conjunto de instrumentos y herramientas orientadas a la creación, fortalecimiento y sostenibilidad de las unidades económicas beneficiarias. Para ello no solo es necesario facilitar el acceso a los recursos, sino también la asesoría en las diferentes áreas que comprometan a la empresa, permitiendo a los individuos descubrir sus capacidades y potenciarlas, con acceso a recursos que antes no tenían, y con conocimientos capaces de adquirir, no solo a través de la experiencia y el error. 
Por otra parte, tal como era de esperar, los microempresarios atendidos por el banco, en general, muestran resultados más favorecedores en comparación con los beneficiarios de la ONG. Estos últimos, en su mayoría, aun acceden a recursos sólo a través de instituciones sin fines de lucro. En este aspecto, aún queda mucho por hacer en términos de las políticas públicas orientadas al sector microempresarial tan diverso, yen término de mayores incentivos para generar una mayor competencia en el sector microfinanciero, complementado con una mayor y más pertinente regulación, lo cual aún es un reto en el mercado microfinanciero chileno como también lo reconoce Economist Intelligence Unit, (2012). Adicionalmente, se requieren estrategias que posibiliten una mayor integración del segmento microempresarial más bajo de la pirámide, sobre todo si lo que se desea es combatir la pobreza generada en las localidades más alejadas de los centros urbanos, y donde principalmente está concentrada la población de menores recursos. Su lejanía, probablemente, es en muchos casos la causa de su exclusión, y no la falta de capacidades y cualidades de emprendimiento. El generar una mayor integración de este colectivo, permitiría, en conjunto con el resto de los emprendimientos, establecer las bases para un desarrollo humano más equilibrado, proporcionando un desarrollo local impulsado desde dentro con el uso de los recursos propios del territorio.

Finalmente, se recomienda para futuras líneas de investigación que puedan llevarse a cabo a partir del mismo, una serie de acciones: evaluar los impactos de otros servicios microfinancieros adicionales al microcrédito, ya sea de forma individual o colectiva; realizar un seguimiento de los beneficiarios de microcrédito y plasmarlo en un estudio longitudinal; aplicar otro tipo de metodologías de selección de grupo control (individuos que hayan postulado para entrar al programa y que no lo hayan hecho aún, individuos que hayan ingresado recientemente, 0 individuos que lleven tiempo participando del programa); y recopilar información sobre otras variables sobre las cuales se quiera determinar impactos, tanto a nivel microempresarial, como familiar e incluso comunitario.

Lo anterior se constituiría en avances que permitirán establecer con mayor confiabilidad la causalidad entre la recepción de servicios microfinancieros y los cambios en la situación de los beneficiarios, aportando a un mejor conocimiento de los efectos que las microfinanzas generan sobre ellos, ya sea en el ámbito económico, social y/o ambiental, y aportando con ello al conocimiento de la relación entre las microfinanzas, las microempresas y el desarrollo local.

\section{REFERENCIAS}

Alburquerque, F. (2002). Desarrollo económico territorial. Guía para agentes. Sevilla: Instituto de Desarrollo Regional, Fundación Universitaria.

Alburquerque, F. (2007). "Desarrollo económico territorial como desafío de política pública". Pp. 69-82. En: Globalización, democracia económica y desarrollo territorial en Chile: Las empresas de menor tamaño en las políticas de Estado. Santiago de Chile: Chile Emprende, SERCOTEC.

Allen, T., Armendáriz, B., Karlan, D. y Mullainathan, S. (2010). Inviting husband in women-only solidarity groups: evidence from southern Mexico. Working Paper. IPA, Harvard University and University College London.

Allende, J. (2000). Medioambiente, ordenación del territorio y sostenibilidad. Bilbao: Universidad del País Vasco.

Angelelli, P., Moudry, R. y Llisterri, J. (2006). Capacidades institucionales para el desarrollo de políticas de fomento de la micro, pequeña y mediana empresa en América Latina y el Caribe. Serie de Informes Técnicos. Washington D.C.: División de la Micro, Pequeña y Mediana Empresa, BID.

Angelucci, M., Karlan, D. and Zinman, J. (2015). Microcredit impacts: evidence from a randomized microcredit program placement experiment by Compartamos Banco. American Economic Journal: Applied Economics, 7(1), 151-182. Disponible en: http://dx.doi.org/10.1257/app.20130537

Attanasio, O., Augsburg, B., De Haas, R., Fitzsimons, E. and Harmgart, H. (2015). The impacts of microfinance: evidence from joint-liability lending in Mongolia. American Economic Journal: Applied Economics, 7(1), 90-122. Disponible en: http://dx.doi.org/10.1257/app.20130489 
Augsburg, B., De Haas, R., Harmgart, H. and Meghir, C. (2015). The impacts of microcredit: evidence from Bosnia and Herzegovina. American Economic Journal: Applied Economics, 7(1), 183-203. Disponible en: http://dx.doi. org/10.1257/app.20130272

Aroca, P. y Hewings, G. (2009). Microcredit impact assessment: the brazilian and chilean cases. Revista Panorama Socioeconómico, 39, 100-112.

Banerjee, A. (2013). Microcredit under the microscope:what do we need to know? Annual Review of Economics, 5, 487-519.

Banerjee, A., Duflo, E., Glennerster, R. y Kinnann, C. (2010). The miracle of microfinance? evidence from a randomized evaluation. Paper. New York: Financial Access Initiative, FAl. Disponible en: http://financialaccess.org/sites/default/ files/The\%20Microfinance\%20Miracle\%20with\%20CP.pdf

Banerjee, A., Karlan, D. and Zinman, J. (2015). Six randomized evaluations of microcredit: introduction and further steps. American Economic Journal: Applied Economics, 7 (1), 1-21. Disponible en: http://dx.doi.org/10.1257/app.20140287

Barnes, C. (1996). Assets and the impact of microenterprise finance programs. Washington, D.C.: Management Systems International.

Bercovich, N. (2004). El microcrédito como component de una política de desarrollo local: el caso del Centro de Apoyo a la Microempresa (CAM), en la ciudad de Buenos Aires. Serie Desarrollo Productivo, 150 (abril). Santiago de Chile: CEPAL.

Berger, M. y Buvinic, M. (1989). Women's Ventures: assistance to the informal sector in Latin America. West Hartford: Kumarian Press.

BID (2005). El grupo BID y la microempresa. Resultados de 15 años de trabajo. Washington D.C.: División de la micro, pequeña y mediana empresa, BID.

Banco Mundial (2008). ¿Finance for all? Policies and pitfalls in expanding access. World Bank Policy Research Report. Washington, D.C.: World Bank.

Berezo, J. (2005). “Las microfinanzas en los países en desarrollo. Pp. 241-282. En: De la Cuesta, Marta y Galindo, Ángel. Inversiones socialmente responsables. Salamanca: Universidad Pontificia de Salamanca.

Boisier, S. (2001). Desarrollo (local) ¿ De qué estamos hablando? En: Vázquez Barquero, Antonio (Comp.). Transformaciones globales, instituciones y políticas de desarrollo local. Rosario: Homo Sapiens Ediciones.

Clark, P. y Kays, A. (1995). Enabling entrepreneurship: microenterprise development in the United Status. New Cork: Aspen Institute.

Coleman, B. (2002). Microfinance in northeast Thailand: Who benefits and how much? ERD Working Paper Series, 9. Manila, Filipinas: Asian Development Bank, Economics and Research Department.

Coleman, B. (2006). Microfinance in northeast Thailand: Who benefits and how much? World Development, 34(9), $1612-1638$. Disponible en: http://www.elsevier.com/wps/find/journaldescription.cws_home/386/description\#description

Comisión Europea (2003). Financiación para el desarrollo local. Nuevas soluciones para acciones público-privada. Dirección General de Empleo y Asuntos Sociales. Traducción y edición en español: DOCUMENTA, Instituto Europeo de Estudios para la Formación y el Desarrollo (Original en inglés, 2001).

Crépon, B., Devoto, F., Duflo, E.,and parienté, W. (2015). Estimating the impact of microcredit on those who take it up: evidence from a randomized experiment in Morocco. American Economic Jornal: Applied Economics, 7(1), 123-150. Disponible en: http://dx.doi.org/10.1257/app.20130535

Crépon, B., Devoto, F., Duflo, E. y Parienté, W. (2011). Impact of microcredit in rural areas of Morocco: Evidence from a randomized evaluation. Working Paper. IPA. Department of Economics, MIT.

Cull, R., Ehrbeck, T. and Holle, N. (2014). Financial inclusion and development: recent impact evidence. Focus Note 92. Washington, DC: Consultative Group to Assist the Poor.

Dini, M. y Stumpo, G. (2018). MIPYMES en América Latina: un frágil desempeño y nuevos desafíos para las políticas de fomento. Documentos de Proyectos (LC/TS.2018/75). Santiago de Chile: Comisión Económica para América Latina y el Caribe (CEPAL).

Duflo, E., Crépon, B., Parienté, W. y Devoto, F. (2008). Poverty, access to credit and the determinants of participation in a new micro-credit program in rural areas of Morocco. Impact Analyses Series, 2. París: Agence Francaise de Développement, AFD; Cambridge: Jameel Poverty Action Lab, J-PAL.

Dunford, C. (2006). Evidence of microfinance contribution to achieving the millennium development goals. Paper for the Global Microcredit Summit, Halifax, Nova Scotia, Canada. EEUU. Freedom from Hunger.

Dunn, E. (2005). Impacts of microcredit on clients in Bosnia and Herzegovina. Impact assessment/Research and Development Component Local Initiatives (Microfinance) Proyect II -LIPII-, presented to Foundation for Sustainable Development of the Federation of Bosnia and Herzegovina and Republika Srpska Development and Employment Foundation.

Economist Intelligence Unit (2012). Microscopio global sobre el entorno de negocios para las microfinanzas 2012. Reino Unido: ElU.

Foschiatto, P. y Stumpo, G. (2006). El microcrédito: un instrumento para fortalecer las capacidades productivas locales. Pp. 21-38. En Foschiatto, Paola y Stumpo, Giovanni (Comp.). Políticas municipales de microcrédito. Un instrumento para la dinamización de los sistemas productivos locales. Estudios de caso en América Latina. Santiago de Chile: Comisión Económica para América Latina y el Caribe, CEPAL.

García, J. (2004). Los micropréstamos como instrumentos de desarrollo en el sur: Especial referencia al ámbito latinoamericano. Madrid: CIDEAL.

Goldberg, N. (2005). Measuring the impact of microfinance: taking stock of what we know. Washington, D.C.: Grameen Foundation USA. 
Gutiérrez, Begoña. (2000). Microcréditos y reducción de la pobreza. La experiencia de la AOD española. Ponencia presentada en VII Jornadas de Economía Crítica, Albacete, España. En: https://webs.ucm.es/info/ec/jec7/pdf/com6-5.pdf

Hulme, D. (2000). Impact assessment methodologies for microfinance: theory, experience and better practice. World Developmnent, 28(1). 79-98. Disponible en: https://doi.org/10.1016/S0305-750X(99)00119-9

Hulme, D. y Mosley, P. (1996). Finance against poverty, 1 y 2. Londres: Routledge.

INE (2015). Síntesis de resultados IV Encuesta de Microemprendimiento. IV EME 2015. Santiago de Chile: INE.

INE (2017). Síntesis de resultados V Encuesta de Microemprendimiento. V EME 2017. Santiago de Chile: INE.

Karlan, D. y Goldberg, N. (2007). Impact evaluation for microfinance: Review of methodological issues. Doing Impact Evaluation, 7. Washington, D.C.: The World Bank.

Karlan, D. y Valdivia, M. (2006). Teaching entrepreneurship: impact of business training on the microfinance clients and institutions. CA, USA: Freedom from Hunger.

Khander, S. (2005). Micro-Finance and poverty: Evidence using panel data from Bangladesh. World Economic Review, $19(2), 263-286$.

Kondo, T. (2007). Impact of microfinance on rural households in the Philippines: a case study from the special evaluation study on the effects of microfinance operations on poor rural households and the status of women. Filipinas: Asian Development Bank, ADB.

Lacalle, M. (2001). Un nuevo instrumento de financiación para luchar contra la pobreza. Revista de Economía Mundial, 5, 121138. Lacalle, M. (2008). Microcréditos y pobreza. De un sueño al Nóbel de la Paz. Madrid: Turpial.

Lacalle, M., Rico, S., Márquez, J. y Durán, J. (2006). Glosario básico de microfinanzas. Cuadernos Monográficos, 5. Madrid: Foro Nantik Lum de Microfinanzas.

Lacalle, M., Rico, S. y Durán, J. (2008). Estudio piloto de evaluación de impacto del programa de microcréditos de Cruz Roja española en Ruanda. Revista de Economía Mundial, 19, 83-104.

Littlefield, E. (2003). Is microfinance an effective strategy to reach the millennium developments goals? Focus Notes 24: CGAP. Martín, J. (2007). Del microcrédito a las microfinanzas. Revista de Empresa, 19, 99-102.

Mballa, L.V. (2017). Desarrollo local y microfinanzas como estrategias de atención a las necesidades sociales: un acercamiento teórico conceptual. Revista Mexicana de Ciencias Políticas y Sociales, 62(229), 101-127. Disponible en: https://doi.org/10.1016/S0185-1918(17)30005-3

McNelly, B. y Dunford, C. (1999). Impact of credit with education on mothers and their young children's nutrition through credit with education program in Bolivia. Research Paper, 5. CA, USA: Freedom from Hunger.

Menon, N. (2003). Consumption smoothing in micro-credit programs. Paper. Departamento de Económicas, Brandesi University.

Metcalfe, M., Gash, M., Gray, B., Reinch, M., Chandler, C. y Dunford, C. (2012). Health and microfinance: leveraging the strenghts of two sectors to alleviate poverty. The Journal of Social Business, 2, 26-44.

Ministerio de Desarrollo Social (2018). Informe de Desarrollo Social 2018. Santiago de Chile: Gobierno de Chile.

Mustafa, S.; Ara, I.; Banu, D.; Hossain, A.; Kabir, A.; Mohsin, M.; Yusuf, A. y Jahan, S. (1996). Beacon of hope: An impact assessment study of BRAC's rural development programme, BRAC. Dacca, Bangladesh: Research and Evaluation Division.

OCDE (2012). Financing SMEs and entrepreneurs 2012: An OECD scoreboard. Paris: OECD Publishing. Disponible en: https://doi.org/10.1787/9789264166769-en

OCDE (2018). Financing SMEs and entrepreneurs 2018: An OECD Scoreboard. Paris: OECD Publishing. Disponible en: https://doi.org/10.1787/fin_sme_ent-2018-en

Odell, K. (2010). Measuring the impact of microfinance: taking another look. Grameen Foundation Publication Series. USA: Grameen Foundation.

Odell, K. (2015). Measuring the impact of microfinance: looking to the future. Grameen Foundation Publication Series. USA: Grameen Foundation.

PNUD (2018). Desigualdad regional en Chile. Ingresos, salud y educación en perspectiva territorial. Santiago de Chile: Programa de las Naciones Unidas para el Desarrollo.

Rico, S. (2009). Microcrédito social: una evaluación de impacto. Barcelona: Fundació Caixa Catalunya.

Rico, S., Lacalle, M., Durán, J. y Márquez, J. (2006). Microempresa y micro-ahorro en la Selva Lacandona, Chiapas: impacto en el progreso social de la mujer indígena. Cuaderno Monográfico, 6. Madrid: Foro Nantik Lum de Microfinanzas.

Rojas, L. (2017). Situación del financiamiento a Pymes y empresas nuevas en América Latina. Santiago de Chile: CIEPLAN.

Rosenberg, R. (2010). ¿El microcrédito ayuda realmente a los pobres? Enfoques, 59. Washington D.C.: CGAP.

Sebstad, J. y Chen, G. (1996). Overview of Studies on the impact of microenterprise credit. Paper submited to USAID by AIMS Project. Washington, D.C.: Management Systems International.

SEEP Network (The) (2001). Aprendiendo de los clientes: herramientas de evaluación para los operadores de microfinanzas. Washington, D.C.: Assessing the Impact of Microenterprise Services (AIMS).

Todd, H. (1996). Women at the center. Grameen bank borrowers after one decade. Dacca, Bangladesh: University Press.

Torres, M. (2006). Microempresa, pobreza y empleo en América Latina y el Caribe: una propuesta de trabajo. Documento para discusión no publicado. Departamento de Desarrollo Social y Empleo, OEA. 
Valdés, J. y Sánchez, G. (2012). Las Mipymes en el contexto mundial: sus particularidades en México. Revista de Ciencias Sociales de la Universidad Iberoamericana, 7(14). 126-156.

Vázquez Barquero, A. (1999). Desarrollo, redes e innovación. Lecciones sobre desarrollo endógeno. Madrid: Ed. Pirámide.

Vázquez Barquero, A. (2013). Desarrollo local, una estrategia para tiempos de crisis. Apuntes del CENES, 28(47), 117-132 Vázquez Barquero, A., y Rodríguez Cohard J.C. (2018). Local development in a global world: challenges and opportunities. Regional Science Policy and Practice. 1-13. Disponible en: https://doi.org/10.1111/rsp3.12164

\section{NOTAS FINALES DEL ARTÍCULO}

' Artículo de investigación desarrollado en Universidad de La Frontera, Temuco, Chile, www.ufro.cl; como continuación del trabajo de Tesis Doctoral en Universidad Autónoma de Madrid, España; y de Proyecto DI16-0113, financiado por la Universidad de La Frontera. Fecha de recepción 06/01/2019. Fecha de aceptación 10/03/2019.

ii Doctora en Integración y Desarrollo Económico, Universidad Autónoma de Madrid, España. Ingeniera Comercial y Licenciada en Ciencias Económicas, Universidad de Concepción, Chile. Académica Jornada Completa del Dpto. de Administración y Economía de la Universidad de La Frontera, Chile. Email: paulina.sanhueza@ufrontera.cl.

iii Estas variables son aquellas en las que se desea similitud entre beneficiarios y controles y aquellas que afectan a la decisión de participar o no en el programa de microcrédito.

iv Para ello, se priorizó solicitar la participación de instituciones de mayor a menor representación regional en términos de cobertura microcrediticia. Al respecto, dos instituciones destacan en el mercado atendido tanto a nivel nacional, como regional: un banco y una ONG.

Este artículo fue editado en la Facultad de Ciencias Administrativas, Económicas y Contables, www.uac.edu.co/facultades/ facultad-ciencias-administrativas-economicas-y-contables, de la Universidad Autónoma del Caribe, www.uac.edu.co, Barranquilla y la Facultad de Administración y Negocios, Universidad Autónoma de Chile, https://www.uautonoma.cl/ facultades_administracion-y-negocios/, Santiago de Chile. 